\title{
Adaptive Data Rate Control for Throughput Improvement and Energy Efficiency in Low Rate WPAN
}

\author{
Indong Yeo \\ Department of Electrical Engineering \\ Korea University \\ Anam-dong, Sungbuk-gu, \\ Seoul, Republic of Korea \\ $+82-2-925-5377$ \\ indongs@dsys.korea.ac.kr
}

\author{
Jonghyune Kim \\ Wireless Systems Architecture Lab. \\ Samsung Electronics \\ Maetan-dong, Suwon City \\ Gyong-gi Province, Republic of Korea \\ $+82-31-279-1114$ \\ hyune.kim@samsung.com
}

\author{
Sunshin An \\ Department of Electrical Engineering \\ Korea University \\ Anam-dong, Sungbuk-gu, \\ Seoul, Republic of Korea \\ $+82-2-925-5377$ \\ sunshin@dsys.korea.ac.kr
}

\begin{abstract}
Energy Efficiency is one of the key issues in Low Rate Wireless Personal Area Networks (LR-WPANs). So far, many research papers have been published on the subject of energy efficiency. However, the throughput in LR-WPAN hasn't been extensively investigated. Therefore, in this paper, we focus on improving the throughput and energy efficiency. LR-WPAN is designed for short range wireless communication based on low power consumption and low cost. In this type of network, the communication capacity may be limited by the low HW specifications such as the small memory size (e.g. network interface buffer) and low processing capacity. In this study, we focus on the former limitation. In this situation, congestion occurs at the Cluster Head $(\mathrm{CH})$ or coordinator owing to its small memory size, which may lead to packet dropping and a reduction of the overall performance. Therefore, we approached this problem from the viewpoint of improving the throughput. Network Devices should consider the congestion situation at the sensor nodes and adopt adaptive data rate control to avoid this situation. The improvement in the throughput and energy efficiency afforded by the adaptive data rate control scheme that is proposed in this paper is confirmed by the network simulation results.
\end{abstract}

\section{Categories and Subject Descriptors}

C.2.1 [Computer Communication Network]: Network Protocols, Wireless Communication - Beacon, Queue, Data Rate; C.4 [Performance of Systems]: Performance Attributes - Throughput

\section{General Terms}

Design, Measurement, Performance

\section{Keywords}

LR-WPAN, 802.15 MAC, Performance, Data Rate, Throughput, Energy

\section{INTRODUCTION}

Basically, LR-WPAN is a wireless communication networking technology for specific applications such as those requiring lowcost and low power. That is, for networks whose main purpose is to process the relevant data that is collected by the network devices and reported to the sink.

Accordingly, LR-WPAN is widely used in fields that require a relatively low data rate and low quality of service. In this type of network, the sensor devices usually operate as an independent RF transceiver without any external power supply. Therefore, they require electric cells or other form of independent power supply. LR-WPAN has the advantages of easy installation and low cost OA\&M (Operation, Administration and Management), so it should have good durability.

For this reason, when we design an LR-WPAN, the most important factor is to operate it with energy efficiency. Nowadays, many wireless communication engineers are studying LR-WPAN from the viewpoint of the energy efficiency.

To solve the problem of excessive energy consumption, two methods can be used.

The first method is to adapt the duty cycle according to the network environment. However, the synchronization problem must be resolved prior to the application of a variable duty cycle. It this problem is not solved, it may lead to a decrease in the network throughput. The second method, the solution is to limit the transmission power at the RF transceiver. However, this scheme requires multi-hop network protocols. And, it may have an implicit weakness that may result in lower throughput. Therefore, we always need to associate the energy efficiency with the throughput when we consider the energy consumption.

As mentioned above, the sensor devices that constitute the LRWPAN have low-cost HW requirements. Consequently, the memory size that is needed by a specific processor and protocol is limited by the specific circumstances. Hence, congestion may be induced by the limited capability or insufficient resources on the receiver side.

When the receiver side is unable to accept or receive packets from the network devices owing to the limited memory size at the network interface of the receiver, the network device that is sending the data adaptively regulates its data rate by receiving feedback messages from the receiver. By means of this mechanism, we can not only prevent unnecessary transmissions causing 
congestion and dropping due to the limited memory, but also obtain an improvement in the throughput and energy consumption. The aim of this study is to control the data rate at the transmitter by receiving feedback information from the receiver. The receiver always monitors its memory of the network interface, which is referred to as a network interface buffer (Queue).

\section{BACKGROUND}

\subsection{Frequency Bands and Data Rate}

Each sensor device in the LR-WPAN can be operated over one or more frequency band. The spreading parameters and data parameters concerning the LR-WPAN are specified in IEEE802.15.4, as shown in Table 1. The LR-WPAN standard supports data rates of 20/40/100/250Kbps.

Table 1. Frequency Bands and Data Rate

\begin{tabular}{|c|c|c|c|c|c|c|}
\hline \multirow{2}{*}{$\begin{array}{c}\text { PHY } \\
\text { (MHz) }\end{array}$} & \multirow{2}{*}{$\begin{array}{c}\text { Frequency } \\
\text { Band } \\
(\mathrm{MHz})\end{array}$} & \multicolumn{2}{|c|}{ Spreading parameters } & \multicolumn{3}{|c|}{ Data parameters } \\
\hline & & \begin{tabular}{|c} 
Chip rate \\
(kchip/sec)
\end{tabular} & Modulation & \begin{tabular}{|l}
$\begin{array}{l}\text { Bit rate } \\
(\mathrm{kb} / \mathrm{sec})\end{array}$ \\
\end{tabular} & $\begin{array}{l}\text { Symbol rate } \\
\text { (ksysmbol/s) }\end{array}$ & Symbols \\
\hline \multirow{2}{*}{$\begin{array}{l}868 \\
/ 915\end{array}$} & $868-868.6$ & 300 & BPSK & 20 & 20 & Binary \\
\hline & $902-928$ & 600 & BPSK & 40 & 40 & Binary \\
\hline \multirow{2}{*}{$\begin{array}{c}868 / 915 \\
\text { (opt) }\end{array}$} & $868-868.6$ & 400 & ASK & 250 & 12.5 & $\begin{array}{l}\text { 20-bit } \\
\text { PSSS }\end{array}$ \\
\hline & $902-928$ & 1600 & ASK & 250 & 50 & $\begin{array}{c}\text { 5-bit } \\
\text { PSSS }\end{array}$ \\
\hline \multirow{2}{*}{$\begin{array}{c}\text { 868/915 } \\
\text { (opt) }\end{array}$} & $868-868.6$ & 400 & O-QPSK & 100 & 25 & $\begin{array}{c}16 \text {-ary } \\
\text { Orthogonal }\end{array}$ \\
\hline & $902-928$ & 1000 & O-QPSK & 250 & 62.5 & $\begin{array}{c}16 \text {-ary } \\
\text { Orthogonal }\end{array}$ \\
\hline 2450 & $\begin{array}{c}2400- \\
2483.5\end{array}$ & 2000 & O-QPSK & 250 & 62.5 & $\begin{array}{c}\text { 16-ary } \\
\text { Orthogonal }\end{array}$ \\
\hline
\end{tabular}

\subsection{Wireless Sensor Network Topology}

LR-WPAN can aid the sensor devices to form a network topology. The main reason for examining the network topology is that it is closely related to the network performance. So, by understanding the formation of the network and the communication between the network devices, we can more easily achieve an appropriate design for applications over the LR-WPAN. We can also better identify implicit problems such as which node is the bottleneck point.

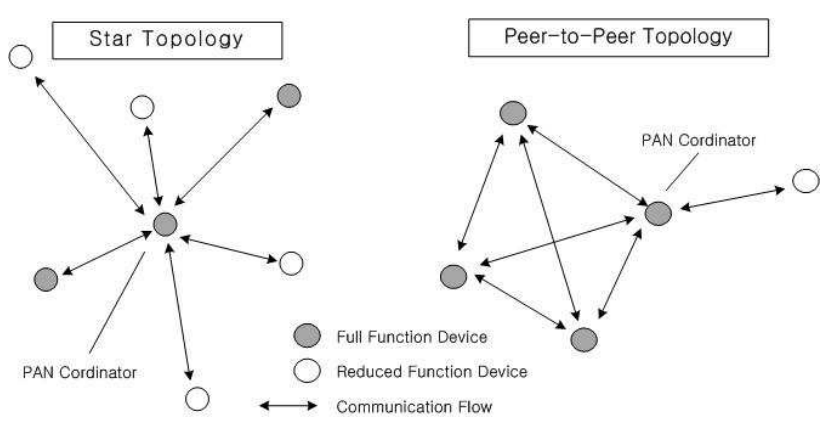

Figure 1. Low Rate WPAN Topology Examples

The star topology is a type of network which is suitable for specific applications that need limited spatial coverage. In the star topology, all of the network devices are arranged centering on the coordinator. In other words, all of the network devices are within the coordinator's coverage area. On the other hand, the peer-topeer topology is appropriate for cluster based networks. Generally, a large network may consist of a few small networks, each having its own $\mathrm{CH}$ or coordinator.

\subsection{Beacon Message}

The main purpose behind the beacon message is to perform network searches. Additionally, it is used for delivering broadcast information. Also, the beacon message is used for network coordination and synchronization such as for the allocation of Guaranteed Time Slots (GTSs). To support the various messages including the beacon message, the LR-WPAN defines 4 types of MAC frame which are Data, ACK, Command and beacon message.

\begin{tabular}{|c|c|c|c|c|c|c|c|c|}
\hline 2 & 1 & 4 or 10 & $\begin{array}{c}0,5,6,10 \\
\text { or } 14\end{array}$ & 2 & $\mathrm{k}$ & $\mathrm{m}$ & $\mathrm{n}$ & 2 \\
\hline $\begin{array}{l}\text { Frame } \\
\text { Control }\end{array}$ & $\begin{array}{c}\text { Sequence } \\
\text { Number }\end{array}$ & $\begin{array}{l}\text { Addressing } \\
\text { Fields }\end{array}$ & $\begin{array}{c}\text { Auxiliary } \\
\text { Security } \\
\text { Header }\end{array}$ & $\begin{array}{l}\text { Super-frame } \\
\text { Specification }\end{array}$ & \begin{tabular}{|c} 
GTS \\
Fields
\end{tabular} & \begin{tabular}{|c} 
Pending \\
Address \\
Fields
\end{tabular} & $\begin{array}{l}\text { Beacon } \\
\text { Payload }\end{array}$ & FCS \\
\hline
\end{tabular}

Figure 2. Schematic View of Beacon Frame

- Super-frame Specification Field : It includes the parameter that describes the super-frame structure.

- Pending Address Specification Field : It includes all the numbers and types in the Address List Field.

- Addressing Field : It includes the list of devices that want to transmit packets to the PAN coordinator

- Beacon Payload Field : It may used when the PAN coordinator has a packet to broadcast over its coverage area.

If a beacon-enabled network is used, the network device first synchronizes with the beacon frame to check whether it is the destination or not.

In this paper, we propose the use of a modified payload field in the beacon message, in order to enable the coordinator to report the congestion of its network interface buffer.

\section{ADAPTIVE DATA RATE DESIGN}

As shown in figure 3, in beacon-enabled LR-WPAN, the network device has to stand by until it receives a beacon message from the PAN coordinator. If the GTS isn't allocated to the network device, it has to transmit its data frames during the contention period according to the CSMA/CA procedure.

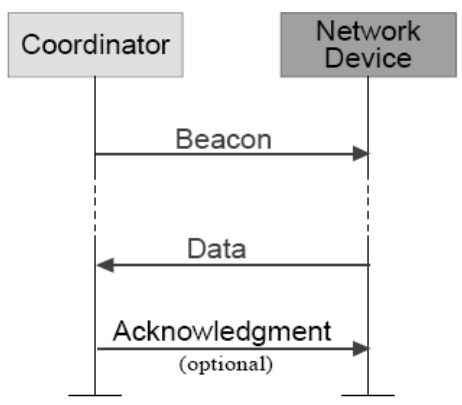

Figure 3. Communication to a coordinator in beaconenabled network 
The main problem which we deal with herein is to transmit the data to the coordinator at a static data rate. If the LR-WPAN allocates slots to the network device using only the RTS/CTS procedure, it brings about congestion in the coordinator, because it doesn't take into account the latter's capacity and capability.

Especially in the case of a cluster-tree network, several CHs may want to transmit data frames from the network device to the coordinator at the same time and, consequently, severe queuing delays may occur in the CH's network interface buffer. Sometimes, this leads to packet dropping. This phenomenon may become worse when a lot of data frames converge into a small number of $\mathrm{CHs}$ and coordinators based on a static data rate.

An increase in the number of dropped packets is not desirable from the viewpoint of the network throughput. Also, dropped packets lead to unnecessary retransmission and inefficient energy consumption. Therefore, in this paper, we propose the use of an adaptive data rate control mechanism by controlling the capacity of the $\mathrm{CH}$ or coordinator according to the current network interface buffer status. Finally, we attempt to prevent the $\mathrm{CH}$ or coordinator from dropping packets from the network devices.

Until now, we described the overall problem. Next, we will provide a more concrete understanding of the packet dropping phenomenon and describe the adaptive data rate control scheme in detail.

At this point, it is necessary to describe the three premises on which the mechanism proposed in this paper is based. First, the network interface buffer of the $\mathrm{CH}$ or coordinator has a maximum storage capacity of $L$ packets. Second, the packet generation and arrival process from each network device to the $\mathrm{CH}$ or coordinator follows a Poisson distribution. Finally, the service time for packets to be transmitted from the network interface buffer of the $\mathrm{CH}$ or coordinator follows a general distribution.

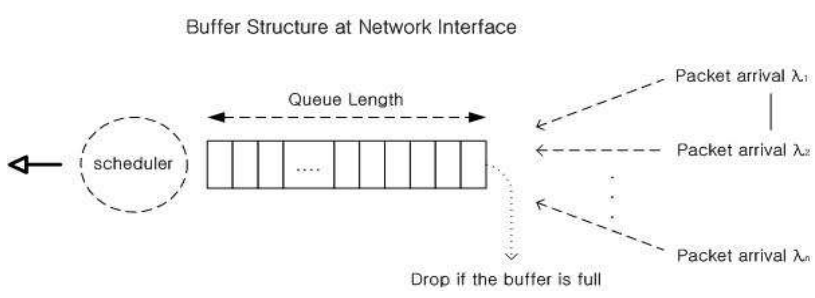

Figure 4. Network Interface Buffer Structure

Consequently, the arrival rate per unit time at the $\mathrm{CH}$ or coordinator can be described by the following equation.

$$
\lambda=\sum_{i=1}^{n} \lambda_{i} \quad(i: i \text { th node }, n: \# \text { of nodes })
$$

As mentioned above, the ultimate purpose of this study is both to avoid the dropping of packets and enhance the channel capacity in the LR-WPAN. To optimize the performance, it is desirable to prevent overflow and underflow at the network interface buffer. When overflow occurs at the network interface buffer, unnecessary transmissions arise which lower the energy efficiency, and this gives rise to a lower throughput.
Allocating a large buffer size may cause an increase in the queuing delay at the network interface buffer, which could result in TCP Retransmission. So, we have to avoid these retransmissions. Given the requirement of low cost for LR-WPAN $\mathrm{HW}$, it is generally preferred to make the buffer size small.

Consequently, it is important not to allow packet dropping to occur in spite of the small size network interface buffer by appropriately adapting the value of $\lambda_{i}$. Therefore, to allow the network devices to support the use of an adaptive data rate, we first have to take into account the delivery capability of the $\mathrm{CH}$ or coordinator to the network devices. As shown in figure 5, the $\mathrm{CH}$ or coordinator always consistently or periodically monitors the amount of packets in its network interface buffer according to the threshold value.

If the number of packets to be serviced is over a certain threshold which is configurable by the operator, the LR-WPAN regards the current state as congestion. At this time, the coordinator has to broadcast a beacon message to all network devices to inform them of this state of congestion.

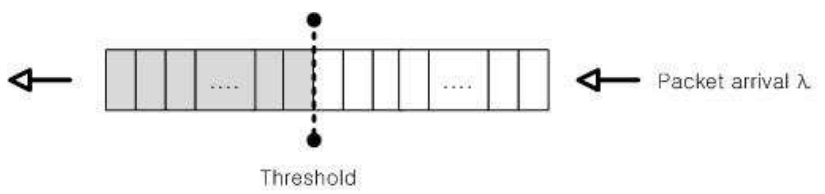

Figure 5. Network Interface Buffer Monitoring

We covered the usage of the beacon message and its field in chapter 2. Currently, we know that the payload field can be defined by the network designer. In this paper, we newly define the Congestion Notification Field $(C N F)$ which occupies one octet in the payload field. Figure 6 shows the proposed payload field in the beacon message.

\begin{tabular}{|c|c|c|c|c|c|c|c|c|c|}
\hline 2 & 1 & 4 or 10 & $\begin{array}{c}0,5,6,10 \\
\text { or } 14\end{array}$ & 2 & $\mathrm{k}$ & $\mathrm{m}$ & 1 & $\mathrm{n}-1$ & 2 \\
\hline $\begin{array}{c}\text { Frame } \\
\text { Control }\end{array}$ & $\begin{array}{c}\text { Sequence } \\
\text { Number }\end{array}$ & $\begin{array}{c}\text { Addressing } \\
\text { Fields }\end{array}$ & \begin{tabular}{|c|} 
Auxiliary \\
Security \\
Header
\end{tabular} & $\begin{array}{l}\text { Super-frame } \\
\text { Specification }\end{array}$ & $\begin{array}{l}\text { GTS } \\
\text { Fields }\end{array}$ & $\begin{array}{c}\text { Pending } \\
\text { Address } \\
\text { Fields }\end{array}$ & CNF & Beacon & FCS \\
\hline
\end{tabular}

Figure 6. Schematic View of Proposed Beacon Frame

The $C N F$ field is set to a value of 1 in the case where congestion occurs at the network interface buffer. Otherwise, the field will be set to 0 . This field may also include the identity of the $\mathrm{CH}$ or coordinator.

$$
P\left\{R_{j+1} \mid R_{j}\right\}=p, \quad P\left\{R_{j-1} \mid R_{j}\right\}=q
$$

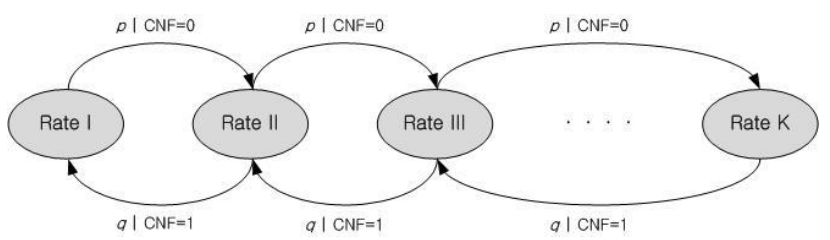

Figure 7. Proposed Data Rate Control Scheme 
According to the $C N F$ value, all of the network devices perform the proposed data rate control scheme described by equation (2), as shown in figure 7 .

Here, $R_{j}$ is the current data rate of a certain network device. If this device receives a beacon message with $C N F$ value $=0$, it has to change its data rate to $R_{j+1}$. Otherwise, if the device receives a beacon message with $C N F$ value $=1$, it has to change its data to $R_{j}$. 1. This control scheme is very simple to implement.

The reason we approach the problem from the viewpoint of random probability parameters $(p$ and $q)$ when we decide the rate is that the network interface buffer is not allocated to a particular network device or its flow. Therefore, we cannot confirm which network device or flow contributes to the congestion. Consequently, the final aim of this paper is to satisfy equation (3).

We attempt to ensure that the transmission data rate of the network device is nearly equal to the arrival rate at the $\mathrm{CH}$ or coordinator. The notation $R_{i}$ is the transmission data rate of the $i$ th network device.

$$
R_{i}=\lim _{t \rightarrow \infty} \lambda_{i}(\text { for } \forall i)
$$

This equation will be almost satisfied when there is little congestion at the $\mathrm{CH}$ or coordinator. Therefore, the value of $\lambda_{i}$ will converge to $R_{i}$.

Next, we will demonstrate the superiority of the proposed method by simulation. By means of simulation, we will confirm the decrease in the number of packets which are dropped and its effect on the throughput and consumed energy.

\section{PERFORMANCE EVALUATION}

\subsection{Simulation Modeling \& Configuration}

Network Simulator version 2 (NS-2) is the most suitable simulator to operate in the UNIX and Linux System environment and it can be applied to wired and wireless network environments. Therefore, it is known as a powerful network simulator.

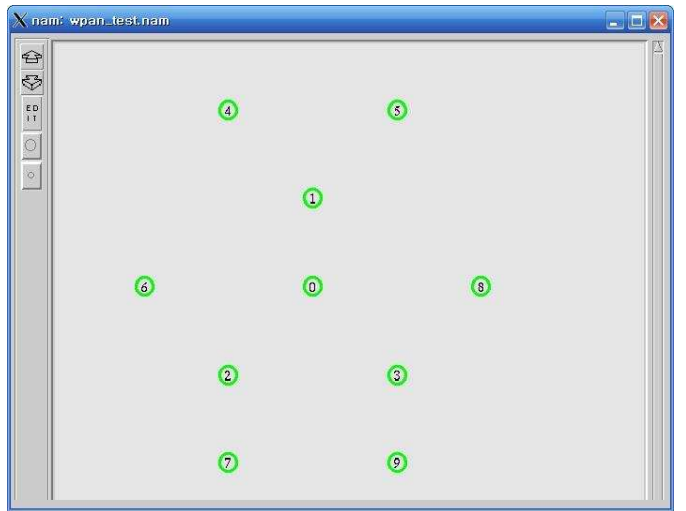

Figure 8. Network Topology Captured by NS-2

Figure 8 shows the configured network topology used to verify our proposed algorithm. Many network devices (Node 4, 5, 6, 7, 8, 9) are already associated with their $\mathrm{CHs}$ (Nodes 1, 2, 3) and the $\mathrm{CHs}$ are also associated with the coordinator (Node 0 ).
For this simulation, we configure the wireless environment parameters and protocols as shown in table 2 .

Table 2. Simulation Configuration Profile for LR-WPAN

\begin{tabular}{|c|c|}
\hline Type & Simulation Environment \\
\hline Base Frequency & $2.4 \mathrm{GHz}$ \\
\hline Channel Model & Wireless / TwoRayGround Model \\
\hline Antenna Type & Omni Directional \\
\hline Link Layer & IEEE802.15.4 MAC + ARP \\
\hline Routing & AODV Routing \\
\hline $\begin{array}{c}\text { Network I/F } \\
\text { Type }\end{array}$ & $\begin{array}{c}\text { Drop-Tail Queue } \\
\text { (Network Interface Buffer) }\end{array}$ \\
\hline
\end{tabular}

The traffic source sends packets to the coordinator following a Poisson distribution. The other parameters related to the traffic pattern are shown in table 3 in detail.

Table 3. Network Configuration and Traffic Pattern

\begin{tabular}{|c|c|}
\hline Type & Configuration \\
\hline Network Topology & Beacon Enable Cluster Tree \\
\hline Number of Nodes & $\begin{array}{c}10 \text { (Coordinator }: 1, \mathrm{CHs}: 3, \\
\text { Network Devices }: 6)\end{array}$ \\
\hline Area (Transmission Range) & $50 \times 50 \mathrm{~m}^{2}\left(15 \mathrm{~m}^{2}\right)$ \\
\hline Number of Flows & Totally 12 Flows \\
\hline $\begin{array}{c}\text { Traffic Generation } \\
\text { Manner }\end{array}$ & $\begin{array}{c}\text { Poisson Manner } \\
(\lambda \fallingdotseq 66.7 \text { per second })\end{array}$ \\
\hline Beacon Mode & $\begin{array}{c}\text { Beacon Order }: 3 \\
\text { Super-frame Order }: 3\end{array}$ \\
\hline
\end{tabular}

Finally, to verify our proposed adaptive data rate mechanism, we introduce some configurable parameters and set their default values as shown in table 4 .

Table 4. Configurable Parameters for Simulation

\begin{tabular}{|c|c|}
\hline Configurable Parameter & Default Values \\
\hline $\begin{array}{c}\text { Queue(Network } \\
\text { Interface Buffer) Size }\end{array}$ & $15,20,25$ \\
\hline $\begin{array}{c}\text { Queue(Network } \\
\text { Interface Buffer) Threshold }\end{array}$ & $50 \%(0.5)$ \\
\hline Probability Parameters $(p, q)$ & $0 \% \sim 70 \%(0 \sim 0.7)$ \\
\hline
\end{tabular}

We allocate a small network interface buffer size in order to verify the reduction in the amount of packet dropping in the worst case. This is the purpose of our simulation. We regard the network interface buffer size as a bottleneck point in the network, and the threshold parameter is used as a linear measure of the congestion at the network interface buffer. Finally, we introduce the probability parameters $(p, q)$ for adaptive data rate control. As mentioned above, when it receives a beacon message with $C N F$, the network device regulates its data rate. However, a rapid 
increase or decrease in the data rate will result in a throughput fluctuation phenomenon. Therefore, we prevent this situation from arising by regulating the $p$ or $q$ parameters.

\subsection{Simulation Results}

First, we configure the network interface buffer size (e.g. queue size) and its threshold. Second, we also vary the probability parameters $(p, q)$, between $0 \%$ to $70 \%$ to increase or decrease the date rate. The simulation results are shown in figures 9, 10, and 11. Firstly, we analyze the throughput in terms of the Goodput in figure 11. In this simulation, the Goodput was about $20 \mathrm{Kbps}$ when $p$ and $q$ were set to $0 \%$, which represents a static data rate. However, in the case where $p$ and $q$ are between $10 \%$ and $30 \%$, the output is $1.2 \sim 1.5$ times the Goodput. Therefore, we can obtain a performance improvement with values of $p$ and $q$ in the range of $10 \% \sim 30 \%$. At this point, we have to consider the energy consumption. If the proposed mechanism were to provide a good performance gain compared to the static data rate mechanism, but with inefficient energy consumption, it would not be satisfactory. However, the results show that the energy consumption is lower than that obtained with the static data rate mechanism. In effect, we can achieve both throughput and energy efficiency with the proposed mechanism.

According to Figure 9, if $p$ and $q$ are over $40 \%$ or $50 \%$, the Goodput decreases below the initial value corresponding to non adaptive data rate control. This is because the strict parameter configuration results in worse throughput. Therefore, we have to optimize the performance by taking into account the network topology.

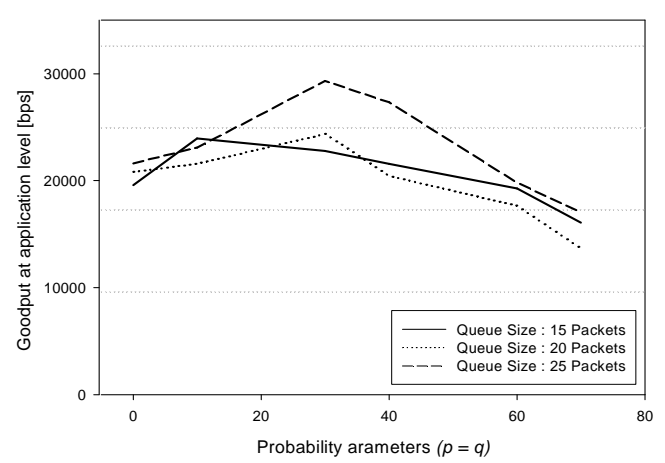

Figure 9. Goodput according to the network interface buffer size \& probability parameters $(p, q)$

Additionally, we calculate the successful transmission ratio according to the probability parameters $(p, q)$, as shown in figure 10.

Successful Transmission Ratio $=\frac{\# \text { successful transmission packets }}{\# \text { total transmission packets }}$

Figure 10 shows that about $70 \% \sim 75 \%$ of the packets successfully arrive at the coordinator from the network device if non adaptive data rate control is applied. However, in the case of the proposed algorithm, over $90 \%$ of the packets successfully arrive at the coordinator from the network device. This result is shown in terms of the successful transmission ratio. This result shows that the number of dropped packets in the network interface buffer of the $\mathrm{CH}$ or coordinator is decreased. Therefore, we would expect the proposed mechanism to improve the energy efficiency.

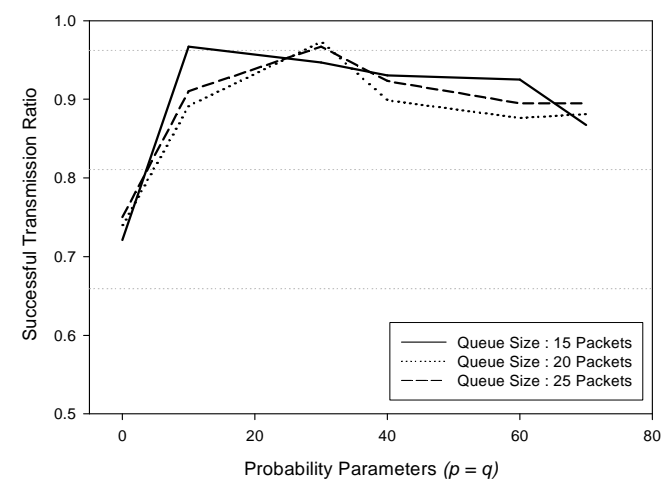

Figure 10. Successful Transmission Ratio

Finally, we estimate the consumed energy using the Energy Model in NS-2. Figure 11 illustrates the consumed energy in our simulation. It is important to mention that a reduction in the consumed energy doesn't necessarily mean an improvement in the performance. If the throughput is decreased along with the energy, it is not good solution. Therefore, the throughput should be kept at the same level or increased while reducing the energy consumption as compared to the previous scheme.

Figure 11 shows that the energy consumption is always less than that in the current LR-WPAN scheme. It is also confirmed that the maximum throughput is obtained in the case where $p$ and $q$ are $10 \% \sim 30 \%$. Now, we can consider both the throughput and energy consumption together.

At the maximum throughput point, we obtain a reduction in the energy consumption compared to the non adaptive data rate scheme.

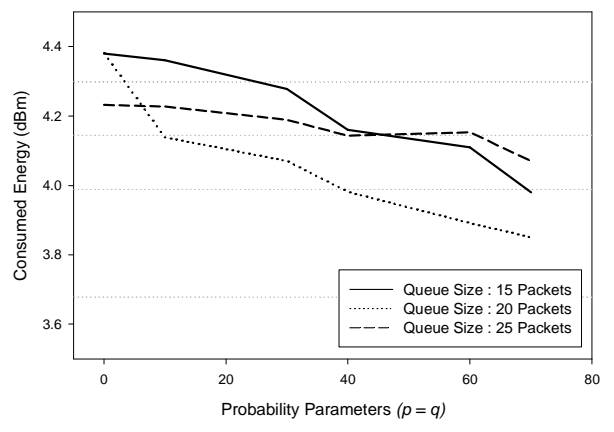

Figure 11. Energy Consumption

This is possible on condition that the configurable parameters is appropriately tuned. According to Figure 11, as the values of $p$ and $\mathrm{q}$ increase, the successful transmission ratio becomes larger. However, at the same time, the Goodput decreases. Consequently, configuring the parameters strictly may not give the best results in terms of the throughput. So far, we have examined the performance of the adaptive data rate algorithm from the viewpoint of the throughput and energy consumption based on the 
NS-2 simulation. In the case of a small allocated network interface buffer size, we can obtain a better throughput by tuning the configurable parameter appropriately.

In the simulation, we obtain an approximately 1.2 1.5 times increase in the throughput and approximately 0.90 0.95 times decrease in the energy consumption at the maximum throughput point over the static data rate mechanism when we configure the parameters as follows :

Param : $p$ and $q=0.1 \sim 0.3(10 \% \sim 30 \%)$, Threshold $=50 \%$

In this study, we included a few scenarios in which the network interface buffer threshold is $50 \%$. However, if we tune the configurable parameter according to the network topology, we will obtain a better throughput for the specific network topology.

\section{CONCLUSION}

Extending the network lifetime by minimizing the energy consumption is one of the most active research fields in LRWPAN.

In this paper, we propose an adaptive data rate mechanism.

This algorithm first considers the network interface buffer capacity of the receiver ( $\mathrm{CH}$ or coordinator) and the receiver monitors the network interface buffer state to determine whether it exceeds the threshold or not.

If the number of packets in the network interface buffer exceeds the threshold, the coordinator broadcasts a new beacon message with a modified payload field to inform the network devices to control their data rate. Through this mechanism, we can improve the performance in terms of both the throughput and energy efficiency. These two factors together allow the network lifetime to be extended.

Through the performance evaluation, we confirmed the validity of the proposed mechanism using the NS-2 simulator and demonstrated its superiority over the current scheme.
We hope that the proposed data rate control mechanism will contribute to the performance enhancement of WPAN.

\section{REFERENCES}

[1] Network Simulator version 2, http://www.isi.edu/nsnam/ns

[2] IEEE Standard 802 Part 15.4 "Wireless Medium Access Control and Physical Layer Specification for Low Rate Wireless Personal Area Networks", IEEE, June 2006

[3] 3GPP2, TSG-C.R1002-0 "cdma2000 Evaluation Methodology Revision 0", 3GPP2, December 2004

[4] Jelena Misic, Shairmina Shafi and Vojislav B. Misic, "Performance of a Beacon Enabled IEEE 802.15.4 Cluster with Downlink and Uplink Traffic", IEEE Transaction on Parallel and Distributed Systems, pp 361-376, 2006

[5] Jelena Misic, Shairmina Shafi and Vojislav B. Misic, "Performance of IEEE 802.15.4 beacon enabled PAN with uplink transmissions in non-saturation mode - access delay for finite buffers", Proceedings of the First International Conference on Broadband Networks (BROADNETS'04),volume 0, pp. 416-425, 2004.

[6] Ranjit Iyer and Leonard Kleinrock, "QoS control for sensor networks," in Proc. ICC'03, May 2003, vol. 1, pp. 517-521.

[7] Gang Lu, Bhaskar Krishnamachari, and Cauligi Raghavendra, "Performance evaluation of the IEEE 802.15.4 MAC for low-rate low-power wireless networks," in Proc. Workshop on Energy-Efficient Wireless Communications and Networks EWCN'04, Apr. 2004.

[8] Hideaki Takagi, "Queuing Analysis, vol. 1: Vacation and Priority Systems", North-Holland, Amsterdam, The Netherlands, 1991 